\title{
Obesity may influence the relationship between sex hormones and lower urinary tract symptoms
}

\author{
Alberto A. Antunes, Luiz Henrique Araújo, Elcio Nakano, Eduardo Muracca, Miguel Srougi \\ Division of Urology, University of Sao Paulo Medical School, SP, Brazil
}

\section{ABSTRACT}

Purpose: The effects of serum testosterone in the lower urinary tract symptoms (LUTS) in patients with benign prostatic hyperplasia (BPH) are not well established. The objective of the study is to evaluate the association of sex hormones with LUTS and control the results by patient weight.

Materials and Methods: The study comprised a cross-sectional analysis of 725 men included in a prostate cancer screening program at University of Sao Paulo Medical School. The serum concentrations of total testosterone (TT), free testosterone (FT) and sex hormone binding globulin (SHBG) were measured. Variables analyzed were age, American Urological Association (AUA) symptom score, storage symptoms, voiding symptoms, quality of life score, prostate specific antigen levels and prostate volume. Obesity was measured through the calculation of body mass index (BMI). A regression analysis model was performed.

Results: Median patient age was 65 years (48 to 94). A higher TT level was significantly associated with a severe AUA symptom score only among patients with a BMI $\geq 25$. Median TT was 371, 370 and 427ng/dL ( $p=0.017$ ) in patients with mild, moderate and severe LUTS respectively. The multivariate regression analysis in patients with BMI $\geq$ 25 showed that only age, TT and sex score were related to LUTS.

Conclusions: A higher TT is associated with a severe AUA score symptom index only in obese patients. Further analysis are necessary to evaluate the mechanisms through which testosterone may influence LUTS in these patients.

\section{ARTICLE INFO}

Key words:

Prostatic Hyperplasia; Lower

Urinary Tract Symptoms;

Testosterone; Obesity

Int Braz J Urol. 2014; 40: 240-6

Submitted for publication:

July 16, 2013

Accepted after revision:

February 29, 2014

\section{INTRODUCTION}

Benign prostatic hyperplasia (BPH) is the main cause of lower urinary tract symptoms (LUTS) in the aging man. About 90\% of men in their 70s have some LUTS related to BPH and the main consequence is impairment in their healthrelated quality of life (1). The lifetime probability among men in their fifties to receive treatment for LUTS secondary to BPH is estimated to reach $35 \%$ of cases (2). Less commonly, LUTS related to BPH may also progress to acute urinary reten- tion, need for surgery, urinary incontinence or recurrent urinary tract infection (3).

Despite these facts, the etiology of BPH is not fully elucidated and several etiologic factors have been proposed (4). Clinical and experimental evidence suggest that testosterone, estrogens and peptide growth factors (GFs) play important roles (5-7). It's well recognized that sexual hormones are essential for normal prostate development and growth. Androgens are also important in the maintenance of $\mathrm{BPH}$ as demonstrated by the size reduction of an enlarged prostate with 
androgen deprivation therapy (8). However, it is unclear whether altered serum concentrations of androgens are associated with increased risk of LUTS or clinical BPH.

Thus far, published studies regarding the association between androgens and LUTS or BPH have reported controversial results (9-12). Additionally, studies that have found positive associations have analyzed different hormones. These discrepancies may be attributed to small sample sizes, important selection bias, inadequate assessment of BPH, and failure to control by other potentially confounding factors that may influence serum testosterone levels, such as obesity $(13,14)$.

As it's well known, obesity in men is commonly accompanied by a decline of serum testosterone (TT) levels through different mechanisms $(15,16)$. The objective of the study was to evaluate the association of sex hormones with LUTS and control the results by the body mass index (BMI).

\section{MATERIALS AND METHODS}

The study comprised a cross-sectional analysis of 725 men who participated in a prostate cancer screening program. Patients who were using 5-alpha reductase inhibitors, alpha adrenergic blockers, had history of prostatic surgery or endocrine diseases, were not considered for analysis. Patients with abnormal digital rectal examination (DRE) findings or an elevated serum PSA level (i.e. $>4.0 \mathrm{ng} / \mathrm{mL}$ ) were referred for prostate biopsy to exclude the possibility of prostate cancer.

Variables analyzed were age, American Urological Association (AUA) symptom score, AUA storage symptoms, AUA voiding symptoms, nocturia, AUA quality of life score (17), international index of erectile function (IIEF) score, prostate specific antigen levels and prostate volume. We measured the serum concentrations of total testosterone (TT), free testosterone (FT) and sex hormone binding globulin (SHBG) in all men. These hormones were measured through the fluorimetric, fluoroimmunoassay, and immunofluorimetric methods respectively (Wallac Perkin Elmer, Finland). We considered the following normal ranges: 271 to $965 \mathrm{ng} / \mathrm{dL}$ for TT, 131 to $640 \mathrm{pmol} / \mathrm{L}$ for FT and 12 to $75 \mathrm{nmol} / \mathrm{L}$ for SHBG. Patients were submitted to DRE, which was performed only by urologists with experience in prostate examination. An enlarged prostate was defined as prostate volume $>30$ grams. Obesity was defined through calculation of the BMI, which was calculated as weight in kilograms divided by the square of height in meters $\left(\mathrm{Kg} / \mathrm{m}^{2}\right)$ and categorized as underweight $\left(<18.5 \mathrm{Kg} / \mathrm{m}^{2}\right)$, normal weight $(18.5$ to $24.9 \mathrm{Kg} / \mathrm{m}^{2}$ ), overweight (25 to $29.9 \mathrm{Kg} / \mathrm{m}^{2}$ ) and obese $\left(\geq 30 \mathrm{Kg} / \mathrm{m}^{2}\right)$. Waist circumference (WC) and hip circumference (HC) were also measured. Demographic characteristics of the men are described in Table-1. Median patient age was 65 years (48 to 94$)$. Median serum PSA level was $1.9 \mathrm{ng} / \mathrm{mL}(0.1$ to 62.6) and median AUA symptom score was 10 (0 to 35). Median total testosterone, free testosterone and SHBG levels were 404.5, 236 and 54ng/ dL respectively. Median BMI was 27 (15 to 48). TT, FT and SHBG were correlated with all clinical parameters. For analysis of obesity, patients were divided in two groups according to the BMI $\geq 25$ or $<25$. Sex hormones were correlated with all urinary symptoms among both groups.

For statistical analysis we used the Chisquare, ANOVA and Kruskal Wallis tests to correlate the sex hormones with clinical parameters. A multivariate regression analysis model was used. Statistical analysis was performed using the SPSS 12.0 for Windows software and significance was set as a $\mathrm{p} \leq 0.05$.

\section{RESULTS}

Table-2 shows correlation of patient characteristics with urinary symptoms. Patients with older age, higher PSA levels and lower IIEF presented worse LUTS with statistically significant results. Patients with a higher TT level and greater prostate weight also presented worse LUTS, but with marginally significant results. Mean TT among patients with mild, moderate and severe symptoms was $430.2,430.3$ and $468.0 \mathrm{ng} / \mathrm{dL}$ respectively. When we analyzed filling symptoms, voiding symptoms and nocturia separately, no statistically significant associations were found.

TT didn't show any significant correlation with the other clinical parameters but with obesity. Patients who were overweight and obese had 
Table 1 - Demographic characteristics of the study population.

\begin{tabular}{lcccc}
\hline Variable & Mean $( \pm \mathrm{SD})$ & Median & Minimun & Maximum \\
\hline Age (years) & $66.0 \pm 9.0$ & 65 & 48 & 94 \\
Total PSA (ng/mL) & $4.6 \pm 7.2$ & 1.9 & 0.1 & 62.6 \\
AUA voiding symptons & $6.3 \pm 6.3$ & 5 & 0 & 20 \\
AUA filling symptoms & $5.9 \pm 4.3$ & 5 & 0 & 15 \\
Nocturia & $2.3 \pm 1.6$ & 2 & 0 & 5 \\
Total AUA score & $12.2 \pm 9.7$ & 10 & 0 & 35 \\
AUA Qol & $2.7 \pm 1.8$ & 3 & 0 & 6 \\
BMI (Kg/m²) & $27.1 \pm 4.2$ & 26.9 & 15.2 & 48.3 \\
Prostate volume (g) & $36.8 \pm 17.9$ & 30 & 15 & 180 \\
TT (ng/dL) & $439.3 \pm 185.1$ & 404.5 & 11.0 & 1332.0 \\
FT (pmol/L) & $242.9 \pm 93.2$ & 236 & 4 & 635 \\
SHBG (nmol/L) & $56.9 \pm 25.6$ & 54 & 10 & 240 \\
\hline
\end{tabular}

Table 2 - Correlation of AUA symptom score categories with clinical variables.

\begin{tabular}{lcccc}
\hline Variable $( \pm \mathrm{SD})$ & Mild & Moderate & Severe & $p$ \\
\hline Age (years) & $64.5( \pm 8.7)$ & $66.4( \pm 9.2)$ & $68.3( \pm 8.6)$ & 0.00 \\
Total PSA $(\mathrm{ng} / \mathrm{mL})$ & $4.7( \pm 9.1)$ & $4.4( \pm 6.7)$ & $7.4( \pm 27.2)$ & 0.01 \\
TT $(\mathrm{ng} / \mathrm{dL})$ & $430.2( \pm 179.5)$ & $430.3( \pm 177.1)$ & $468.0( \pm 202.9)$ & 0.06 \\
FT $(\mathrm{pmol} / \mathrm{L})$ & $239.8( \pm 97.2)$ & $241.6( \pm 88.7)$ & $250.2( \pm 92.7)$ & 0.48 \\
SHBG $(\mathrm{nmol} / \mathrm{L})$ & $56.8( \pm 24.4)$ & $55.7( \pm 27.4)$ & $58.9( \pm 25.0)$ & 0.45 \\
IIEF & $16.4( \pm 7.5)$ & $14.1( \pm 7.6)$ & $11.7( \pm 7.6)$ & 0.00 \\
BMI $\left(\mathrm{kg} / \mathrm{m}^{2}\right)$ & $27.1( \pm 4.0)$ & $27.0( \pm 4.3)$ & $27.3( \pm 4.5)$ & 0.72 \\
WC $(\mathrm{cm})$ & $95.0( \pm 11.6)$ & $96.3( \pm 13.1)$ & $95.8( \pm 13.0)$ & 0.35 \\
HC $(\mathrm{cm})$ & $99.2( \pm 9.3)$ & $98.1( \pm 14.7)$ & $98.7( \pm 10.2)$ & 0.59 \\
Prostatic Weight $(\mathrm{g})$ & $36.2( \pm 0.0)$ & $40.7( \pm 2.0)$ & $41.6( \pm 10.0)$ & 0.07 \\
\hline
\end{tabular}

lower levels of TT $(\mathrm{p}<0.001)$. Median FT levels were statistically lower among patients with 65 years or older (211 vs $254 \mathrm{ng} / \mathrm{dL})(\mathrm{p}<0.001)$, and with a higher BMI $(\mathrm{p}=0.006)$. SHBG levels were statistically higher among patients with 65 years or older ( $<$ 0.001) and statistically lower among patients with a higher BMI. These two hormones failed to show any significant correlation with urinary symptoms or other clinical parameters (data not shown).

Due to associations between serum hormone levels and surrogate measures of obesity, 
we analyzed the associations between hormone levels and urinary symptoms among patients with BMI $<25$ (underweight or normal weight) and $\geq$ 25 (overweight or obese) separately. The results showed that TT levels were significantly associated with AUA symptom score among patients with a BMI $\geq 25$. Median TT was 371,370 and $427 \mathrm{ng} / \mathrm{dL}$ $(\mathrm{p}=0.017)$ in patients with mild, moderate and severe LUTS respectively. FT and SHBG were not associated with urinary symptoms in this BMI group (Table-3). Analysis of voiding symptoms, filling symptoms and nocturia separately failed to show any association with hormone levels according to this BMI category.

Table 3 - Logistic regression analysis for the occurrence of severe LUTS among patients with $B M I \geq 25$.

\begin{tabular}{lccc}
\hline Variable $( \pm \mathrm{SD})$ & OR & $95 \% \mathrm{Cl}$ & $\mathrm{p}$ \\
\hline Age (years) & 1.03 & $1.00-1.06$ & 0.02 \\
Total PSA $(\mathrm{ng} / \mathrm{mL})$ & 1.00 & $0.99-1.02$ & 0.23 \\
TT $(\mathrm{ng} / \mathrm{dL})$ & 1.00 & $1.00-1.00$ & 0.00 \\
IIEF & 0.95 & $0.93-0.98$ & 0.00 \\
WC $(\mathrm{cm})$ & 0.90 & $0.34-2.38$ & 0.83 \\
Prostatic Weight $(\mathrm{g})$ & 1.00 & $0.99-1.01$ & 0.64 \\
\hline
\end{tabular}

No associations were found among patients with a BMI $<25$. Median TT was 453, 450 and $467 \mathrm{ng} / \mathrm{dL}$ ( $\mathrm{p}=0.952$ ) among patients with mild, moderate and severe LUTS respectively. FT and SHBG were not associated with urinary symptoms in this BMI group. Analysis of voiding symptoms, filling symptoms and nocturia separately also failed to show any association.

Finally, we performed a multivariate regression analysis for the occurrence of severe LUTS among patients with a BMI $\geq 25$ and $<25$. An increased age, a higher TT level and a worse IIEF were independently associated with the presence of severe LUTS among patients from the former group. Only a worse IIEF was independently associated with LUTS among patients with a BMI $<25$.

\section{DISCUSSION}

In the present study, we found that serum TT and FT levels were significantly lower, and SHBG levels were significantly higher in obese patients when compared to patients with lower weights. When the entire cohort was analyzed, TT levels were higher in patients with severe LUTS, however, these results reached only marginal statistical significance. When patients with a BMI $\geq 25$ were analyzed, TT levels were significantly higher in patients with severe LUTS when compared to patients with moderate or mild LUTS. These findings suggest that sexual hormones may play a role in the pathogenesis of LUTS related to BPH. Multivariate analysis confirmed TT as an independent variable associated to a severe LUTS. No correlations were observed among patients with a BMI $<25$.

The reasons why sex hormones may influence LUTS related to BPH are not well described. Despite the lack of knowledge regarding several aspects of BPH pathogenesis, its well recognized that the development of the histological features of the disease is dependent on the bioavailability of TT and its metabolite, dihydrotestosterone (18). A congenital lack of $5 \alpha$-reductase results in a vestigial prostate gland (19) and castration in a man leads to glandular atrophy and regression of LUTS (20). It's also reasonable to think that systemic effects of TT in bone and muscle mass, fat mass, body energy, and physical, sexual, and cognitive function may also be reflected on LUTS. Finally, there may be some direct effects of such hormones on bladder muscle function.

Despite these facts, most studies to date have reported inverted or no associations between sex hormones and LUTS. Trifiro et al. (10) evaluated baseline serum sex hormone concentrations and subsequent risk of LUTS with 20-year followup in a cohort of 158 community-dwelling older men. In opposition to our findings, they demonstrated that men with higher mid-life levels of testosterone/dihydrotestosterone and bioavailable testosterone had a decreased 20-year risk of LUTS. There were no significant associations of TT, oestradiol, testosterone/oestradiol, dihydrotestosterone, or dehydroepiandrosterone with LUTS. 
Some limitations were the small sample size, the homogenous character of the community cohort (mostly white and upper middle class) limiting the external validity, and the 20-year gap between hormone measurement and LUTS assessment that precluded analysis of changes in serum sex steroid levels during this period. In another study, the authors performed a nested case-control analysis using the placebo arm of the Prostate Cancer Prevention Trial. The authors reported a decreased risk of incident BPH with higher baseline total testosterone and total testosterone : 17 $\beta$-diolglucuronide (21). Litman et al. (9), analyzing data from the Boston Area Community Health (BACH) Survey, reported an inverse correlation of AUASI with both TT and bioavailable testosterone in non-age adjusted models and bioavailable testosterone in age-adjusted models. No significant association between LUTS and FT were found in the present analysis. Interestingly, some authors have also reported inverse relationships between LUTS and FT levels $(22,23)$.

Conversely, other studies have reported positive relationships. Favilla et at. (2010) investigated a possible association between the severity of LUTS and the serum levels of sex hormones in 122 men with symptomatic BPH. In accordance to our results, on statistical analysis they found that the total IPSS was significantly associated with age and TT but not with free testosterone or the serum levels of the other sex hormones. While men with a IPSS $>19$ presented a median testosterone of $425.6 \mathrm{ng} / \mathrm{mL}$, median testosterone level among men with a IPSS $<19$ was $346.8 \mathrm{ng} / \mathrm{mL}$ ). However, the limitations of the study were again the small sample size and the fact that they only included men with severe LUTS who were candidates to surgery.

Reasons that may help to explain the lack of associations in some studies may be attributed to determination of sexual hormones levels at different points in the natural history of BPH. Additionally, it's possible that plasma levels of these hormones are not representative of intra-prostatic levels. Regarding this fact, the dosage of sexual hormones metabolites may be more representative of the intra-prostatic environment. Platz et al. (12), demonstrated the association between an SHBG metabolite, androstanediol glucuronide (AAG), severe LUTS and the risk of BPH surgery. Patients with higher serum levels of AAG were at increased risk of having either BPH surgery or severe LUTS.

The unique strength of the present analysis is that we demonstrated a clear influence of BMI on the relationship between testosterone and LUTS, a variable that has been neglected by most studies. According to some reports, obesity may be associated with BPH. Giovannucci et al. (24), followed men enrolled in the Health Professionals Follow-up Study for the incidence of prostatectomy due to BPH and for the frequency and severity of symptoms of urinary obstruction. After adjustment for age, smoking, and BMI, only abdominal obesity showed a relationship with the rate of prostatectomy. Similar results were observed regarding frequency of urinary symptoms among those without prostatectomy (OR 2.0). However, these results should be analyzed carefully, since only a minority of patients with BPH undergoes surgical treatment for relieving LUTS.

Conversely, associations between obesity and BPH were not reproduced by other authors. Meigs et al. (25), defined risk factors for a clinical diagnosis of BPH among subjects of the population-based Massachusetts Male Aging Study. They found that BMI did not individually predict clinical BPH. More recently, in the study of 1,206 participants in the comparison arm of the Air Force Health Study with a median follow-up of 15.6 years, no relation was seen between weight or BMI and BPH (26). In another recent study of a cohort of healthy caucasian men aged 40-79 years randomly selected from the Olmsted County, Minnesota, few significant associations of anthropometric measures with the presence or progression of components of BPH were found (27).

Clearly, most of the controversy regarding relationship between $\mathrm{BPH}$ and anthropometric measures is due to the variable definitions of $\mathrm{BPH}$ used in studies. Technically, the diagnosis of BPH is made based on histological examination of a prostate specimen from biopsy, surgery or autopsy. However, as this approach gives limited insight into the incidence or progression of the disease, studies have considered surrogate measures to as- 
sess BPH, including clinical (LUTS), physiologic (urinary flow rates), anatomic (prostatic volume) and biochemical (PSA levels) measures (28). For this reason, in the present study we investigated specifically the associations with LUTS.

Some advantages of the present study over the others should be mentioned. We analyzed a relative large series of patients who were not candidates for surgery, thus all LUTS categories could be analyzed. Additionally, patients were not using any placebo, which could have influenced LUTS. Limitations of the present study should also be noted. We didn't analyze other important sex hormones such as dihydrotestosterone and oestradiol. Other clinical conditions that could influence sex hormone levels such as metabolic syndrome, diabetes or tobacco use were not included in the analysis.

Finally, if the results of the present series could be confirmed by further clinical analyses, there will be a role for the clinical use of serum testosterone, at least in obese patients with BPH. Basic research studies addressing the mechanisms of action of testosterone over the bladder and prostate tissues on a molecular basis are also necessary for the understanding of LUTS physiopathology and for the development of new therapeutic targets.

\section{CONCLUSIONS}

Serum TT and FT levels are significantly lower, and SHBG levels is significantly higher in obese patients when compared to patients with lower weights. A higher TT is independently associated with a worse AUA symptom index only in obese patients.

\section{ABBREVIATIONS}

LUTS = Lower Urinary Tract Symptoms

BPH = Benign Prostatic Hyperplasia

TT $=$ Total Testosterone

FT $=$ Free Testosterone

AUA = American Urological Association

$\mathrm{BMI}=$ Body Mass Index

GFs $=$ Growth Factors

DRE $=$ Digital Rectal Examination

SHBG $=$ Sex Hormone Binding Globulin

\section{CONFLICT OF INTEREST}

\author{
None declared.
}

\section{REFERENCES}

1. Walsh PC: Treatment of benign prostatic hyperplasia. N Engl J Med. 1996; 335: 586-7.

2. Oesterling JE: Benign prostatic hyperplasia: a review of its histogenesis and natural history. Prostate Suppl. 1996; 6: 67-73.

3. Cockett AT, Barry MJ, Holtgrewe HL, Sihelnick S, Williams $\mathrm{R}, \mathrm{McC}$ nnell J: Indications for treatment of benign prostatic hyperplasia. The American Urological Association Study. Cancer. 1992; 70: 280-3.

4. Guess HA: Benign prostatic hyperplasia: antecedents and natural history. Epidemiol Rev. 1992; 14: 131-53.

5. Steiner MS: Review of peptide growth factors in benign prostatic hyperplasia and urological malignancy. J Urol. 1995; 153: 1085-96.

6. Soulitzis N, Karyotis I, Delakas D, Spandidos DA: Expression analysis of peptide growth factors VEGF, FGF2, TGFB1, EGF and IGF1 in prostate cancer andbenign prostatic hyperplasia. Int J Oncol. 2006; 29: 305-14.

7. Smith P, Rhodes NP, Ke Y, Foster CS: Upregulation of estrogen and androgen receptors modulate expression of FGF-2 and FGF-7 in human,cultured, prostatic stromal cells exposed to high concentrations of estradiol. Prostate Cancer Prostatic Dis. 2002; 5: 105-10.

8. Peters CA, Walsh PC: The effect of nafarelin acetate, a luteinizing-hormone-releasing hormone agonist, on benign prostatic hyperplasia. N Engl J Med. 1987; 317: 599-604. Erratum in: N Engl J Med. 1988; 318: 580.

9. Litman HJ, Bhasin S, O'Leary MP, Link CL, McKinlay $\mathrm{JB}, \mathrm{BACH}$ Survey Investigators: An investigation of the relationship between sex-steroid levels and urological symptoms: results from theBoston Area Community Health survey. BJU Int. 2007; 100: 321-6.

10. Trifiro MD, Parsons JK, Palazzi-Churas K, Bergstrom J, Lakin $\mathrm{C}$, Barrett-Connor E: Serum sex hormones and the 20-year risk of lower urinary tract symptoms in community-dwelling oldermen.BJU Int. 2010; 105: 1554-9.

11. Platz EA, Kawachi I, Rimm EB, Longcope C, Stampfer MJ, Willett WC, et al.: Plasma steroid hormones, surgery for benign prostatic hyperplasia, and severe lower urinary tractsymptoms. Prostate Cancer Prostatic Dis. 1999; 2: 285-289.

12. Favilla V, Cimino S, Castelli T, Madonia M, Barbagallo I, Morgia G: Relationship between lower urinary tract symptoms and serum levels of sex hormones in men withsymptomatic benign prostatic hyperplasia. BJU Int. 2010; 106: 1700-3. 
13. Selvin E, Feinleib M, Zhang L, Rohrmann S, Rifai N, Nelson WG, et al.: Androgens and diabetes in men: results from the Third National Health and Nutrition Examination Survey(NHANES III). Diabetes Care. 2007; 30: 234-8. Erratum in: Diabetes Care. 2007; 30: 1683.

14. Li C, Ford ES, Li B, Giles WH, Liu S: Association of testosterone and sex hormone-binding globulin with metabolic syndrome and insulin resistance in men. Diabetes Care. 2010; 33: 1618-24.

15. Couillard C, Gagnon J, Bergeron J, Leon AS, Rao DC, Skinner JS, et al.: Contribution of body fatness and adipose tissue distribution to the age variation in plasma steroidhormone concentrations in men: the HERITAGE Family Study. J Clin Endocrinol Metab. 2000; 85: 1026-31.

16. Gautier A, Bonnet F, Dubois S, Massart C, Grosheny C, Bachelot $A$, et al:: Associations between visceral adipose tissue, inflammation and sex steroid concentrations in men. Clin Endocrinol (0xf). 2013; 78: 373-8.

17. Barry MJ, Fowler FJ Jr, O’Leary MP, Bruskewitz RC, Holtgrewe $\mathrm{HL}$, et al.: The American Urological Association symptom index for benign prostatic hyperplasia. The MeasurementCommittee of the American Urological Association. J Urol. 1992; 148: 1549-57; discussion 1564.

18. Bartsch G, Rittmaster RS, Klocker H: Dihydrotestosterone and the concept of 5alpha-reductase inhibition in human benign prostatic hyperplasia. Eur Urol. 2000; 37: 367-80.

19. Imperato-McGinley J, Guerrero L, Gautier T, Peterson RE: Steroid 5alpha-reductase deficiency in man: an inherited form of male pseudohermaphroditism. Science. 1974; 186: 1213-5.

20. Huggins C, Clark PJ: Quantitative studies of prostatic secretion: II. The effect of castration and of estrogeninjection on the normal and on the hyperplastic prostate glands of dogs. J Exp Med. 1940; 72: 747-62.

21. Kristal AR, Schenk JM, Song Y, Arnold KB, Neuhouser ML, Goodman PJ, et al.: Serum steroid and sex hormonebinding globulin concentrations and the risk of incident benign prostatichyperplasia: results from the prostate cancer prevention trial. Am J Epidemiol. 2008; 168: 1416-24.
22. Miwa Y, Kaneda T, Yokoyama 0: Association between lower urinary tract symptoms and serum levels of sex hormones in men. Urology. 2008; 72: 552-5.

23. Tan MO, Karabiyik I, Uygur MC, Diker Y, Erol D: Serum concentrations of sex hormones in men with severe lower urinary tract symptoms and benign prostatic hyperplasia. Int Urol Nephrol. 2003; 35: 357-63.

24. Giovannucci E, Rimm EB, Chute CG, Kawachi I, Colditz GA, Stampfer MJ, et al.: Obesity and benign prostatic hyperplasia. Am J Epidemiol. 1994; 140: 989-1002.

25. Meigs JB, Mohr B, Barry MJ, Collins MM, McKinlay JB: Risk factors for clinical benign prostatic hyperplasia in a community-based population of healthy agingmen. J Clin Epidemiol. 2001; 54: 935-44.

26. Gupta A, Gupta S, Pavuk M, Roehrborn CG: Anthropometric and metabolic factors and risk of benign prostatic hyperplasia: a prospective cohort study of Air Force veterans. Urology. 2006; 68: 1198-205.

27. Burke JP, Rhodes T, Jacobson DJ, McGree ME, Roberts R0, Girman CJ, et al.: Association of anthropometric measures with the presence and progression of benign prostatichyperplasia. Am J Epidemiol. 2006; 164: 41-6.

28. Jacobsen SJ, Girman CJ, Lieber MM: Natural history of benign prostatic hyperplasia. Urology. 2001; 58: 5-16; discussion 16. 